\title{
evolución de las instalaciones de cocción en las fábricas de cemento de Norteamérică
}

A. S. KaNNEWURF y C. F, CLAUSEN «Rock Products», mayo 1962, pág. 128.

Durante la última década, la industria del cemento norteamericana ha sufrido una modernización y una expansión sin precedentes. Siguiendo la línea de unidades de producción mayores con una eficiencia mejorada, los nuevos hornos se han hecho más largos y más anchos.

¿En qué extensión este desarrollo ha alterado el aspecto de la industria y cuál es el estado presente del equipo y elementos auxiliales en el departamento de hornos? Para contestar a esta pregunta se ha hecho recientemente una revisión de las instalaciones industriales que abarca 133 fábricas de cemento distribuidas entre los EE. UU. y Canadá. Los hornos comprendidos en este estudio totalizan 577. Prohablemente sea la encuesta más amplia de este tipo, y los datos contenidos en ella proporcionan la respuesta a muchas cuestiones. Aun cuando no se dispone de todos los datos, la información recogida y analizada servirá como base para estudios adicionales en las instalaciones industriales de hornos.

Un aumento en el volumen del horno no siempre significa un aumento de producción, aun cuando el volumen sea uno de los factores más importantes.

Este hecho es evidente a partir de los datos recogidos que muestran una relación entre el volumen del horno y la producción. Asimismo, la dispersión de producción entre hornos de igual tamaño no puede explicarse basándose exclusivamente en la limitada información obtenida en este trabajo. Por ejemplo, dos de los hornos referidos son idénticos de tamaño, pero uno produce $135 \mathrm{t}$ por día $\mathrm{y}$ el otro 261 t. La capacidad de equipo auxiliar tal como molinos de carbón y ventiladores de tiro limita la producción lo mismo que el volumen del horno. Otro factor importante, aunque intangible, es las variaciones en la marcha del proceso de una empresa a otra o de una fábrica a otra.

Se han dado un gran número de formas de producción de los hornos, y se ha comprobado que ninguna de ellas es exacta en todas las conclusiones. El número de variables que interviene es muy grande, aunque en general, sin embargo, pueden darse fórmulas aproximadas para la producción basándose en las dimensiones del horno. Más adelante se presentan dos tipos de fórmulas: uno basado en el volumen del horno y otro tanto en el volumen como en la superficie. Ambas se encuentran entre las fórmulas más exactas de que se dispone para predecir la producción media de un horno.

Las fórmulas de producción basadas en el volumen del horno (todos los cálculos se basan en el diámetro interior libre) se obtuvieron de la flgura 1 , y se derivaron de dos tipos de producción media. Una para hornos de via seca con un volumen inferior a 10.000 pies cúbicos y la otra para todos los hornos de vía húmeda y dos de vía seca con volumen superior a 10.000 pies cúbicos:

Para hornos de vía seca con volumen inferior a 10.000 pies cúbicos:

Producción (barriles $/$ dia $)=96+0,128$ volumen (pies cúbicos).

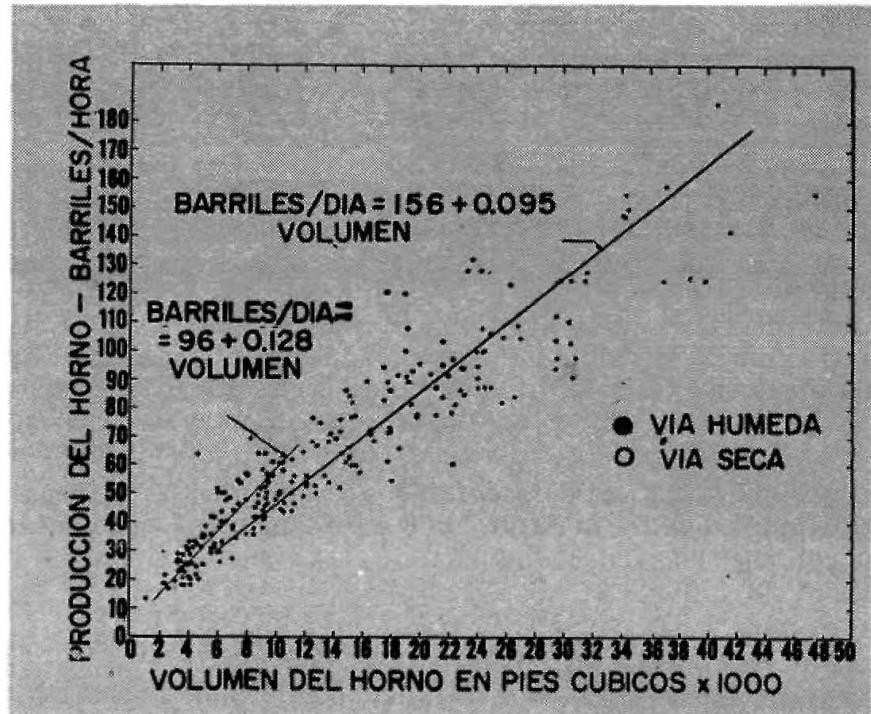

Fig. 1. Producción del horno en relación al volumen del mismo. 
Para todos los hornos de via húmeda y los de via seca con volumen superior a t0.000 pies cúbicos:

Producción (barriles/dia) $=156+0,095$ volumen (pies cúbicos).

La clave para los cálculos que siguen es el factor adimensional volumen-superficie (V S), calculado sumando el $35 \%$ del volumen (pies cúbicos) con el $65 \%$ de la superficie (pies cuadrados). Su origen es la formula Kuhl-Smidth modificada, que tiene su origen en la industria de construcción de maquinaria:

Para hornos de via seca con un $\mathrm{V} S$ inferior a 7.000 :

Producción (barriles $/$ dia) $=0,22 \mathrm{~V}$ s.

Para todos los hornos de vía húmeda $\mathrm{y}$ los de via seca con un $\mathrm{V} S \mathrm{~S}$ superior a 7.000 :

$\mathrm{V} \mathrm{s}$.

Producción $\quad($ barriles $/$ dia $)=200+0,142$

El efecto del contenido de humedad en el enudo de alimentación sobre la producción es muy pronunciado. A ello se deben las diferencias en Ias fórmulas antes expuestas; una es para los hornos de via seca más cortos y la otra para los de vía húmeda más largoss.

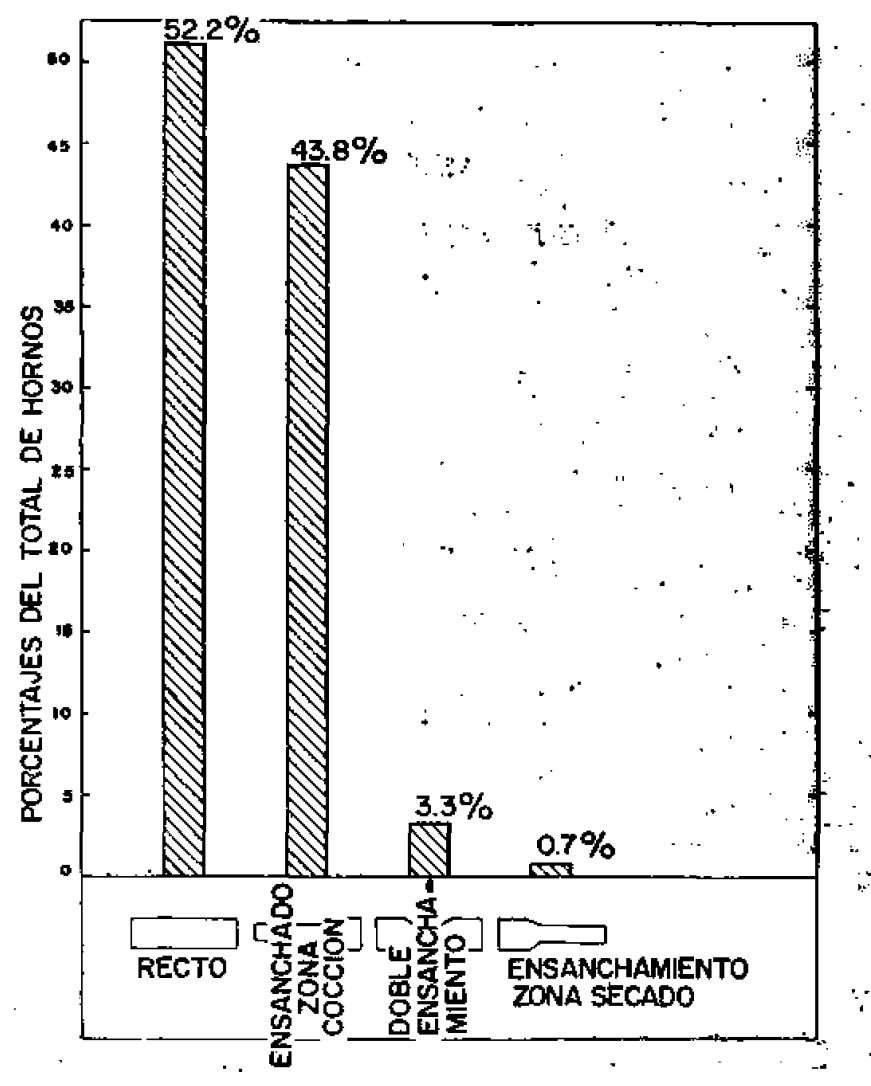

Fig. 2. Forma del horno.

Puede hacerse una nueva distinción de los hornos de via húmeda, desglosándolos en alto y bajo contenido de humedad. La producción de los hornos que alimentan con bajo contenido de humedad en el crudo (filtros) se representa mejor por la fórmula:

Producción (barriles $/$ dfa $)=120+0,153$ V s.

Todas las causas de esta alta velocidad de producción no pueden atribuirse a los filtros, porque gran parte de los hornos con filtro están equipados con calderas de recuperación de calor. En muchos casos que mantienen las temperaturas de los gases de escape lo bastante altas para producir una energia efectiva, es esto más responsable de la alta producción que los filtros. Además, el uso de filtros de vacio que producen una acostran mejora el movimiento de caida del material con ventajas en Ia transmisión de calor entre los gases calientes y el refractario, por una parte, y el material, por la otra.

De esta discusión, parece natural suponer que los hornos largos de via seca tendrán una capacidad de producción mayor que los de via húmeda del mismo tamaño. Este no es siempre el caso, porque muehos de elios no son mejores que la media de los hornos de vía húmeda. Una razón podría ser que las pérdidas de polvo en los hornos de via seca son muy grandes en las altas producciones. Otra razón podria ser que los hornos de via húmeda más largos tienen espacio para una transmisión de calor en 1a zona de secado más adecuada, compensando asi las ventajas de la alimentación seca. También se ha sugerido que la alimentación en seco, cuando está caliente, tiende a fluir como si fuera, un líquido con poco efecto de cascada, lo cual puede ser la razón para que los grandes hornus de via seca tengan sólo un poco más de capacidad de producción que los hornos de via húmeda de igual longitud.

En la figura 2 se representan las cuatro formas básicas de hornos y el porcentaje instalado de cada uno de ellos. Los hornos con doble ensanchamiento presentan en la mayor parte de los casos una producción superior a la media.

El objeto primordial de las modificaciones a través de los años ha sido la reducción en el consumo de combustible. Los precalentadores en via seca y los sistemas de filtros y cadenas en via húmeda han jugado un papel excelente en este sentido, como lo demuestra el consumo de combustible más bajo registrado en este trabajo: $980 \mathrm{kcal} / \mathrm{kg}$. 
El consumo medio de calor para hornos de via seca era $1.850 \mathrm{kcal} / \mathrm{kg}$, con un máximo de 2.560 y un mínimo de 970 . Para los hornos de vía húmeda era de $2.070 \mathrm{kcal} / \mathrm{kg}$, con un máximo de $2.610 \mathrm{y}$ un minimo de 1.435 .

Si se representan Btu/hora frente al volumen del horno se obtienen resultados similares a los de la figura 1 (producción/volumen del horno). Esto indica que la producción depende del volumen del horno y de la cantidad de calor que es posible introducir en el mismo.

La figura 3 representa las necesidades de combustible por bbl. de clínker, las cuales disminuyen al aumentar el volumen del horno.

En la tabla I se señalan los tipos y combinaciones de combustibles y el número de hornos que usa cada uno de ellos.

TABLA I.-Combustibles.

\begin{tabular}{lrrrr} 
& \multicolumn{3}{c}{ Número de hornos } \\
Tipo de combustible & Via húmeda & Vla seca & Total \\
Carbón. . . . . . . . & 132 & 246 & 378 \\
Gas y carbón . . . . . & 32 & 39 & 71 \\
Gas . . . . . . . . & 40 & 11 & 51 \\
Fuel-oil . . . . . . & 23 & 10 & 33 \\
Fuel-0il y gas. . . . . & 5 & 18 & 23 \\
Fuel-oil y carbón . . . & 2 & 7 & 9 \\
Gas, carbón y fuel-oil . & 3 & 4 & 7 \\
Sin información . . . & 5 & 0 & 5 \\
\hline
\end{tabular}

El número de hornos instalados cada cinco años se representa en la figura 4. Se clasifican según el proceso (seco o húmedo) y se ve claramente que hace cincuenta años los hornos de vía seca dominaban en la industria. Gradualmente, la vía húmeda inclina la balanza en su favor. Desde 1935, sin embargo, la vía seca volvió a adquirir valor, y hoy día le supera de nuevo en las más recientes instalaciones.

En la figura 5 se representa el volumen de hornos instalados en períodos de cinco años. Si se compara con la figura 4 puede verse que, aunque el número de hornos instalados en los últimos cinco años incluidos en esta estadística no es tan grande como en otros períodos previos, el volumen instalado es, sin embargo, el mayor, debido a la tendencia hacia hornos mayores.

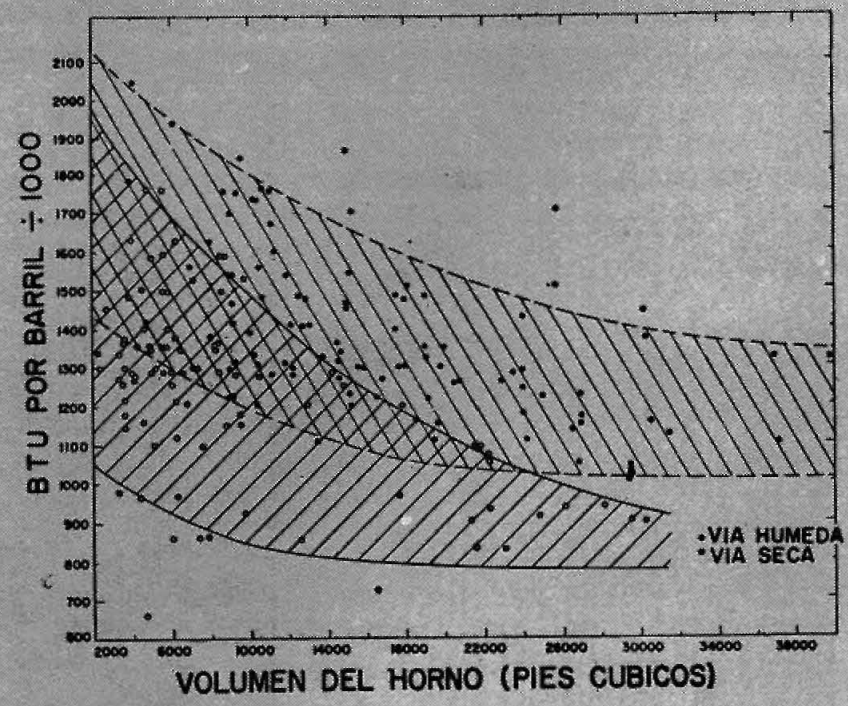

Fig. 3. Btu por barril consumidas en relación con el volumen del horno.

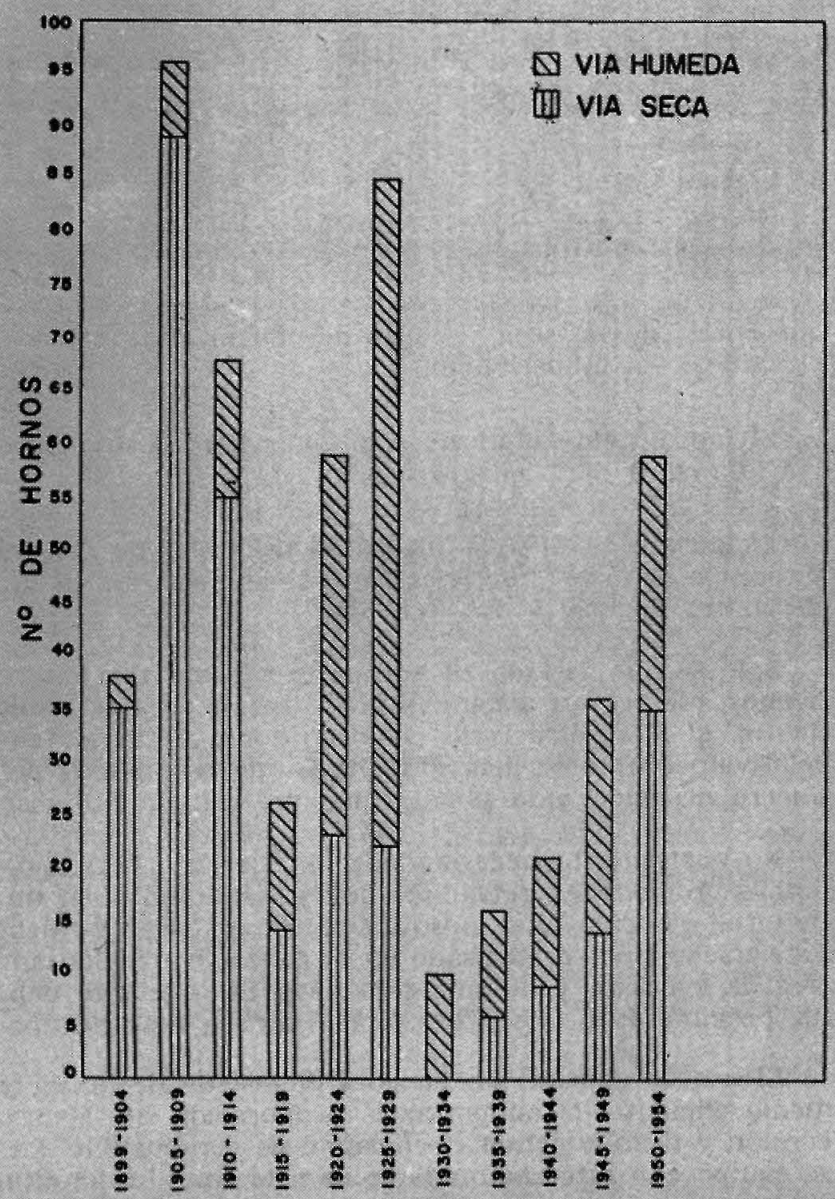

Fiz. 4. Hornes instalados en períodos de cinco anios (1899-1954). 


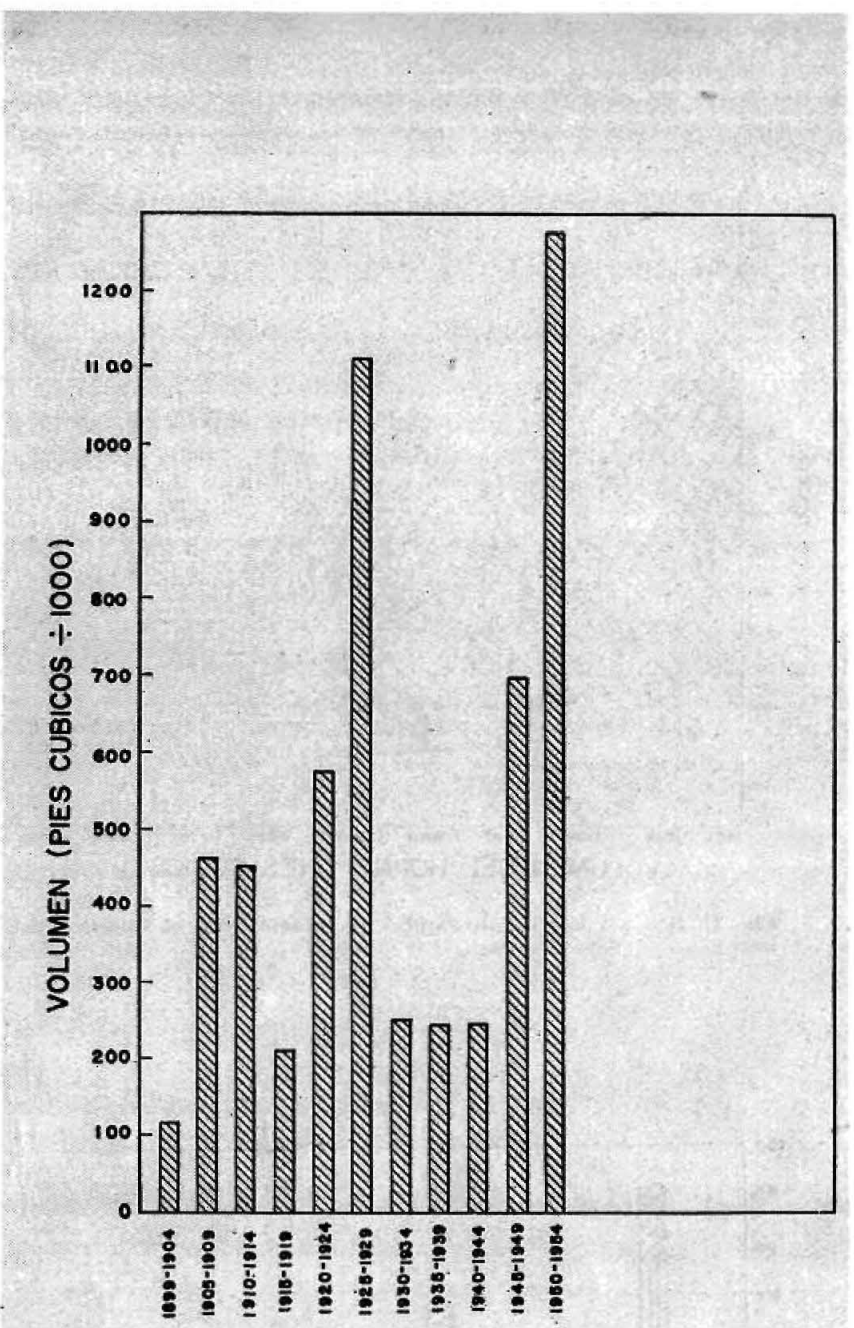

Fig. 5. Volumen total de hornos instalados en períodos de cinco años (1899-1954)
Las longitudes de los hornos oscilan de 18 a $153 \mathrm{~m}$. Una comparación de la distribución de tamaños entre los hornos de 1936 y 1955 demuestra que los hornos cortos se han sustituido por hornos largos. Por ejemplo, en 1936 habia 115 hornos de $35 \mathrm{~m}$ de longitud. En 1955 este número bajó a 74, en tanto que el número de hornos de $106 \mathrm{~m}$ subió de 4 a 16.

La vida fel revestimiento refractario es el factor que decide, en la mayor parte de los casos, el tiempo que un horno de cemento puede marchar sin interrupciones.

En este trabajo, el horno se divide en cuatro zonas: descarga, cocción, calcinación y precalentamiento y secado. El ladrillo aluminoso $\left(<50 \% \quad \mathrm{Al}_{2} \mathrm{O}_{3}\right)$ predomina en la zona de precalentamiento tanto en vía seca como en vía húmeda.

El ladrillo extraaluminoso redujo el empleo del aluminoso en la zona de calcinación de los hornos de vía húmeda del $63 \%$ al $35 \%$. En las zonas de calcinación de vía seca se redujo el consumo del extraaluminoso del $75 \%$ al $24 \%$.

El ladrillo básico se usó en el $60 \%$ de las zonas de cocción de vía húmeda frente al $37 \%$ con ladrillos extraaluminosos. Esta relación se invirtió en zonas de cocción de vía seca donde se empleó ladrillo extraaluminoso en el $70 \%$ de los casos y básicos en el $17 \%$. Una posible razón de esto es que las condiciones en la zona de cocción de los hornos de vía húmeda son más severas que en los de vía seca, a causa del mayor consumo de calor por $\mathrm{kg}$ de clínker requerido para evaporar el agua de la alimentación.

El ladrillo extraaluminoso ha sido el más empleado en la zona de descarga: $62 \%$ de los casos en vía húmeda y $56 \%$ en vía seca.

El ladrillo de sílice se utilizó en las zonas de descarga y cocción de 18 hornos de vía seca. Los revestimientos de hormigón refractario se encuentran en la zona de secado de nueve hornos de vía seca y de cuatro hornos de vía húmeda.

Sólo se han aislado 26 hornos (ver tabla II). Este es un paso hacia atrás, porque en 1936 había 44 hornos que tenían aislamiento. La razón de esta caída brusca en el uso de este material es, probablemente, que el refractario colocado sobre él tiene tendencia a caer después de un tiempo de trabajo relativamente corto. Las dificultades de reemplazar el revestimiento sobrepasaron los beneficios del ahorro de calor, y la popularidad del aislamiento disminuyó rápidamente.

No obstante, la necesidad del aislamiento persistió, y la última novedad del caso ha sido combinar ambas propiedades, refractariedad y aislamiento, en un único revestimiento. La denominación de este material «ladrillo aluminoso aislante expuesto», no debe confundirse con forro aislante o aislamiento. Este nuevo tipo se ha usado en la zona de precalentamiento y secado de cinco hornos de vía húmeda y tres de vía seca, y aunque sólo haya de momento ocho casos en este artículo, la opinión es que, en un próximo futuro, se revestirán muchos más hornos con este material.

El uso de cadenas en la zona de secado de los hornos de vía húmeda se ha reconocido como un medio eficiente de aumentar la evaporación del agua del crudo, arrastrando éste hacia la zona de cocción y disminuyendo el consumo de combustible. La mayoría de los hornos de vía húmeda $(61,3 \%)$ se equipó con intercambiadores de este tipo. La longitud media de una zona de cadenas es de $16,35 \mathrm{~m}$, abarcando aproximadamente el $20 \%$ de la longitud del horno, y la distancia media del extremo de alimentación al principio de la zona de cadenas es de 4,75 metros. 
TABLA II.-Aislamiento del horno.

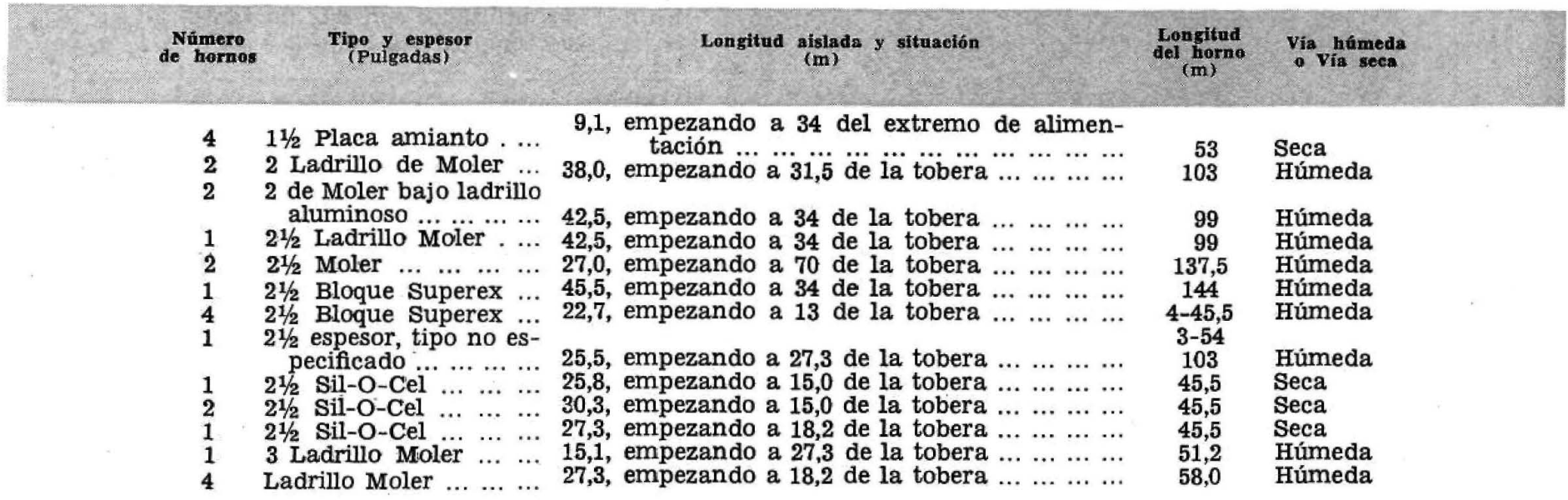

El sistema de cadenas pesa un promedio de $15.700 \mathrm{~kg}$. El mayor peso total de cadenas registrado en cualquier horno era de $61.800 \mathrm{~kg}$ en una zona de 16,70 $\mathrm{m}$ de longitud y 4,05 m de diámetro.

La forma más popular de unión es la elíptica: $75 \%$; el $23 \%$ de forma de ocho; el $2 \%$, circular, y el $1 \%$, combinación de circular y elíptica.

El $75 \%$ de todos los sistemas de cadenas estaban construidos con material de $5 / 8$ de pulgada; el $21 \%$, de $3 / 4$ de pulgada, y el $3 \%$, una mezcla de $5 / 8$ y $3 / 4$ de pulgada. El $1 \%$ restante usaba, o bien cadena de 1 pulgada, o bien una combinación de cadenas de $5 / 8$ y $1 / 8$ de pulgada.

El sistema espiral de suspensión de cadenas se usó en 85 hornos; el de cortina, en 9 hornos, y una combinación de ambos sistemas, en 4 hornos. Todos ellos se suponen con densidad simple. Independientemente del método de suspensión, se registran dos hornos con una sección de cadenas de densidad 1/2, cuatro con densidad 2 y tres con una combinación de densidades sencilla y doble.

Para cierto número de hornos se utilizan las áreas por unidad de longitud de varios tamaños de cadena para calcular la superficie total de cadenas expuesta.

La superficie de cadenas parece aumen tar linealmente, en un amplio intervalo, con el-volumen del horno. Sólo un horno, que se equipó con filtros y cadenas, tenia un sistema de cadenas corto; el interés fundamental es transportar el material, más que actuar como cambiador de calor.

La colección de polvo se usó en más del $66 \%$ de los hornos revisados en este trabajo. El $48 \%$ de los sistemas instalados son colectores mecánicos (fig. 6); los sistemas eléctricos llegan al $39,1 \%$. La combinación de ambos se emplea en 9,7\% de los hornos con colectores, en tanto que el 2,9\% utilizó la sedimentación en húmedo.

El polvo recogido en el $91 \%$ de los hornos con colectores volvió al sistema de un modo $\mathrm{u}$ otro.

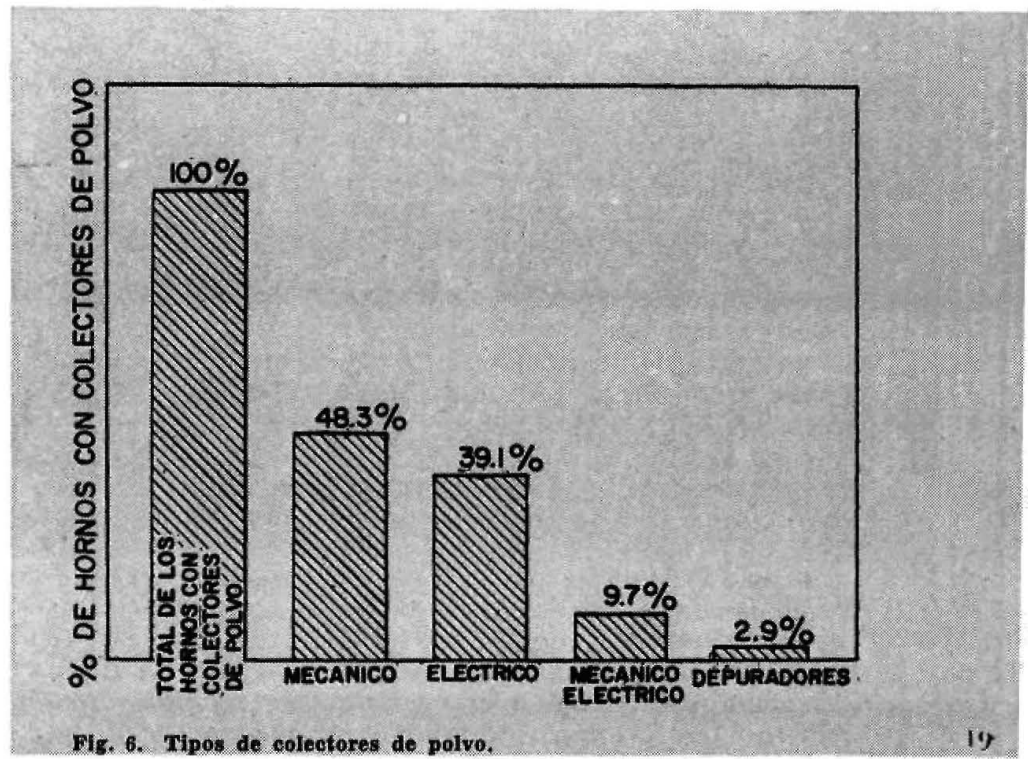




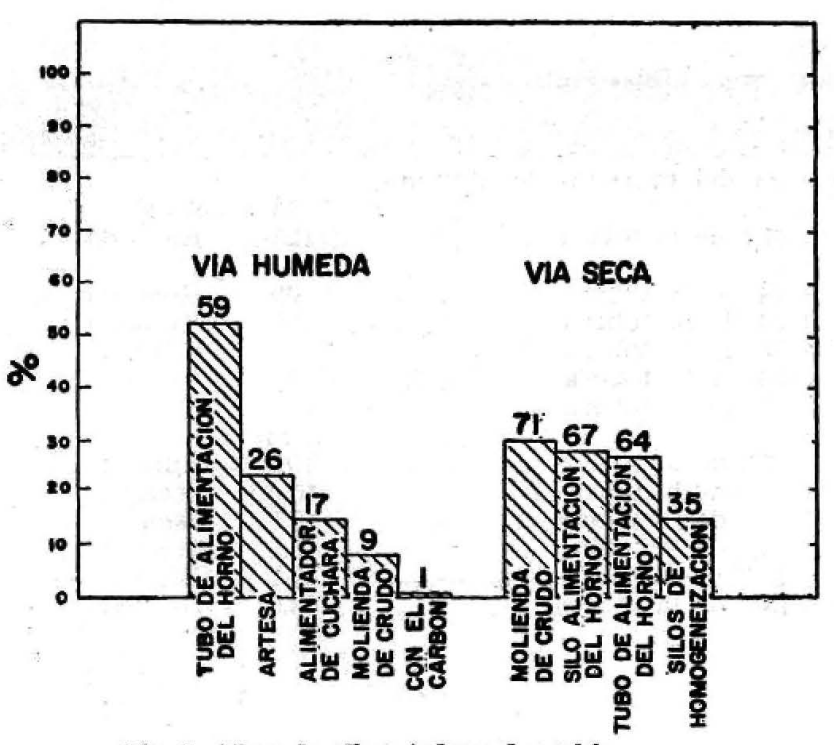

Fig. 8. Tipos de alimentadores de carb6n.

TABLA III-AAlímentadores de horno.

TIPO DE ALIMENTADOR

Núm. de hornos alimentados

Vía húmeda Vía seca

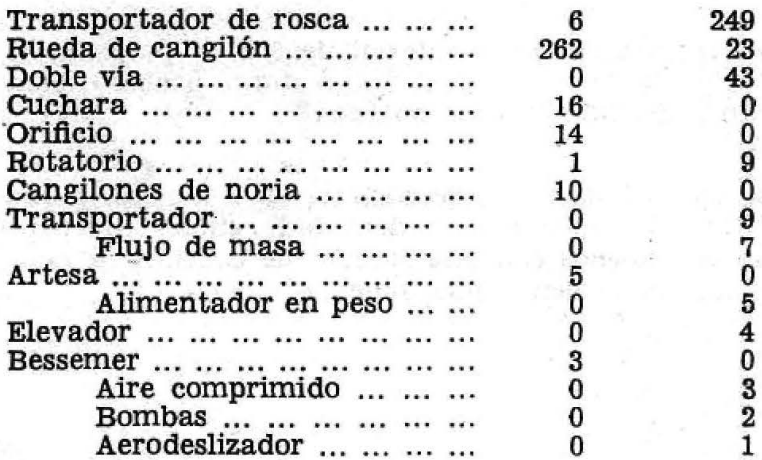

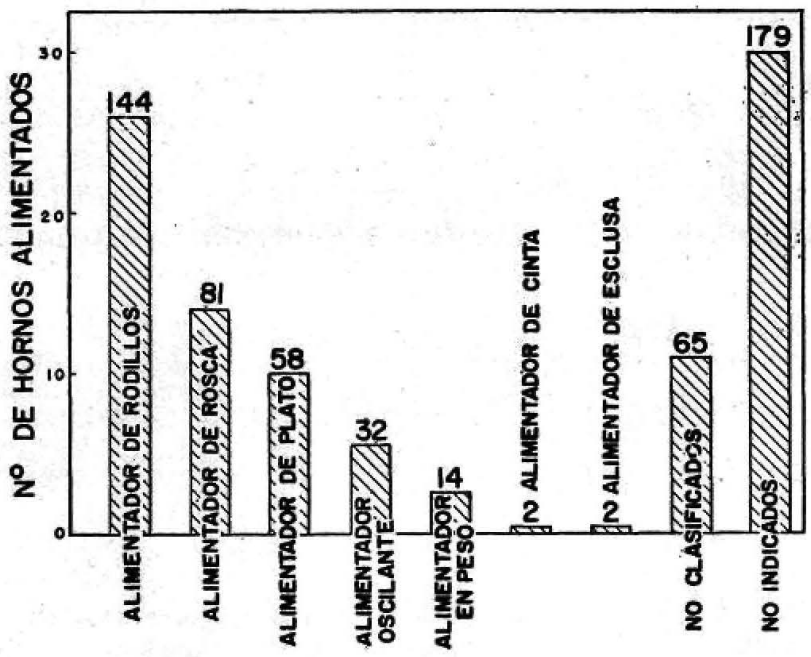

Fig. 7. Punto de retorno del polvo al horno.

En la figura 7 se hace un desglose de los métodos utilizados con este fin tanto en vía seca como en via húmeda.

El peso medio de polvo recogido por tonelada de clínker era de $106 \mathrm{~kg}$ en hornos de via húmeda y $128 \mathrm{~kg}$ en hornos de vía seca.

El número y tipos de alimentadores empleados, tanto en vía seca como en via húmeda, se indica en la tabla III. En ella se demuestra que la cinta transportadora para hornos de vía seca y la rueda de cangilón para vía húmeda destacan con mucho sobre el total de los otros alimentadores empleados.

La sincronización del alimentador con el horno tuvo lugar por medios mecánicos en el $39 \%$ de los casos. La sincronización manual se llevó a cabo el $28 \%$ de las veces y la eléctrica en el $27 \%$ de los casos.

La mayor parte de los filtros de horno se instalaron entre 1927 y 1932 , época en que las calderas de recuperación alcanzaron su máxima popularidad.

De todos, 53 hornos en 22 plantas emplearon alimentación filtrada y la mayoría de ellos $(90,5 \%)$ también tenían instaladas calderas de recuperación de calor. De los 53 hornos, 43 empleaban filtros de discos y 10 filtros de tambos. Los filtros redujeron el contenido medio de humedad de la pasta de 36,8 a $20,4 \%$, lo que supone reducir el $45 \%$ la cantidad de agua evaporable. Los hornos que utilizan filtros son todos relativamente cortos, siendo la longitud media $52 \mathrm{~m}$ y la mayor 90 metros.

Ocho hornos llevaban precalentadores de alimentación, cinco en vía húmeda y tres en vía seca. Los cinco de vía húmeda eran precalentadores de pasta instalados delante de la filtración. Dos de los instalados en vía seca son del tipo de suspensión Humboldt, y el tercero, es un precalentador Grudex.

Los sistemas de combustión del carbón para hornos se dividian en dos grupos: sistemas con silos y alimentación directa. La última es más moderna y popular, ya que 295 de los hornos relatados (el $62 \%$ ) empleaban sistemas de este tipo.

La temperatura media de entrada de aire en las unidades de molienda era de $214^{\circ} \mathrm{C}$ y la de salida de $80^{\circ} \mathrm{C}$. La cantidad del aire de combustión total que pasa por las unidades de molienda era del orden del $29 \%$, con un $5 \%$ de valor minimo y $62 \%$ de máximo registrados. 
TABLA IV.-Relación longitud de horno/potencia motor (HP).

$\begin{array}{lcccccccccc}\text { Longitud horno }(\mathrm{m}) & <30,5 & 30,5-45,5 & 45,5-60,5 & 60,5-76 & 76-91 & 91-106 & 106-121 & 121-136 & 136-151 \\ \begin{array}{l}\text { Número de hornos } \\ \begin{array}{l}\text { Longitud horno (m)/mo- } \\ \text { tor (HP) }\end{array}\end{array} & 23 & 43 & 31 & 21 & 10 & 22 & 19 & 11 & 3 \\ & 1,30 & 1,11 & 1,09 & 0,92 & 0,93 & 1,13 & 1,10 & 1,01 & 0,77\end{array}$

Las ligeras diferencias de finura del carbón registradas en los dos sistemas son las siguientes:

SISTEMA CON SILO ALIMENTACION DIRECTA

$\%$ que pasa por 100 mallas

$\%$ que pasa por 200 mallas
Media: 91,2

Intervalo: 75,3 a 97,9

Media: 80,5

Intervalo: 47 a 93
Media: 93,6

Intervalo: 75 a 99

Media: 72

Intervalo: 65 a 96

El número y tipo de alimentadores de carbón en uso durante los períodos indicados en este trabajo se indican en la figura 8.

El enfriador de clínker más popular-con mucho-era el de parrilla, que incluye tanto el tipo horizontal como el inclinado. Se introdujo rápidamente en el mercado en los primeros siguientes años a 1930, posiblemente como resultado del trabajo hecho por la P. C. A. sobre la importancia del enfriamiento rápido para reducir la expansión de los cementos al autoclave y la expansión a largo plazo de los hormigones. También se supone que tiene un efecto beneficioso sobre la molturabilidad del clínker. Desde entonces, el número de instalaciones que han adoptado este sistema se extiende a casi la mitad de los hornos en funcionamiento (ver figura 9). La temperatura media a que sale el clínker en este enfriador es de $77^{\circ} \mathrm{C}$, la cual no es la media más baja entre todos los tipos de enfriadores.

Le siguen los enfriadores rotatorios, aunque desciende continuamente el número de los instalados. Abarcan el $25,8 \%$ de los incluidos en este trabajo. La temperatura media a que sale el clínker en estos enfriadores es de $127^{\circ} \mathrm{C}$.

Los recuperadores abarcan el $9,6 \%$ del total, y casi la mitad de ellos están combinados o bien con el enfriador vertical o bien con el rotatorio. La temperatura media de salida del clínker en los recuperadores es de $500^{\circ} \mathrm{C}$, que en un caso bajó a $81^{\circ} \mathrm{C}$ cuando el recuperador se instaló en serie con un enfriador rotatorio.

Los enfriadores verticales ocupan el cuarto lugar con el $9,2 \%$ de las instalaciones totales y tienen la temperatura media de enfriamiento del clínker más baja con $75^{\circ} \mathrm{C}$. Sin embargo, son de capacidad más bien baja e inadecuados para usar con los grandes hornos modernos de gran producción. La última instalación de este tipo se hizo en 1928, y es sorprendente encontrar todavía 44 en funcionamiento.

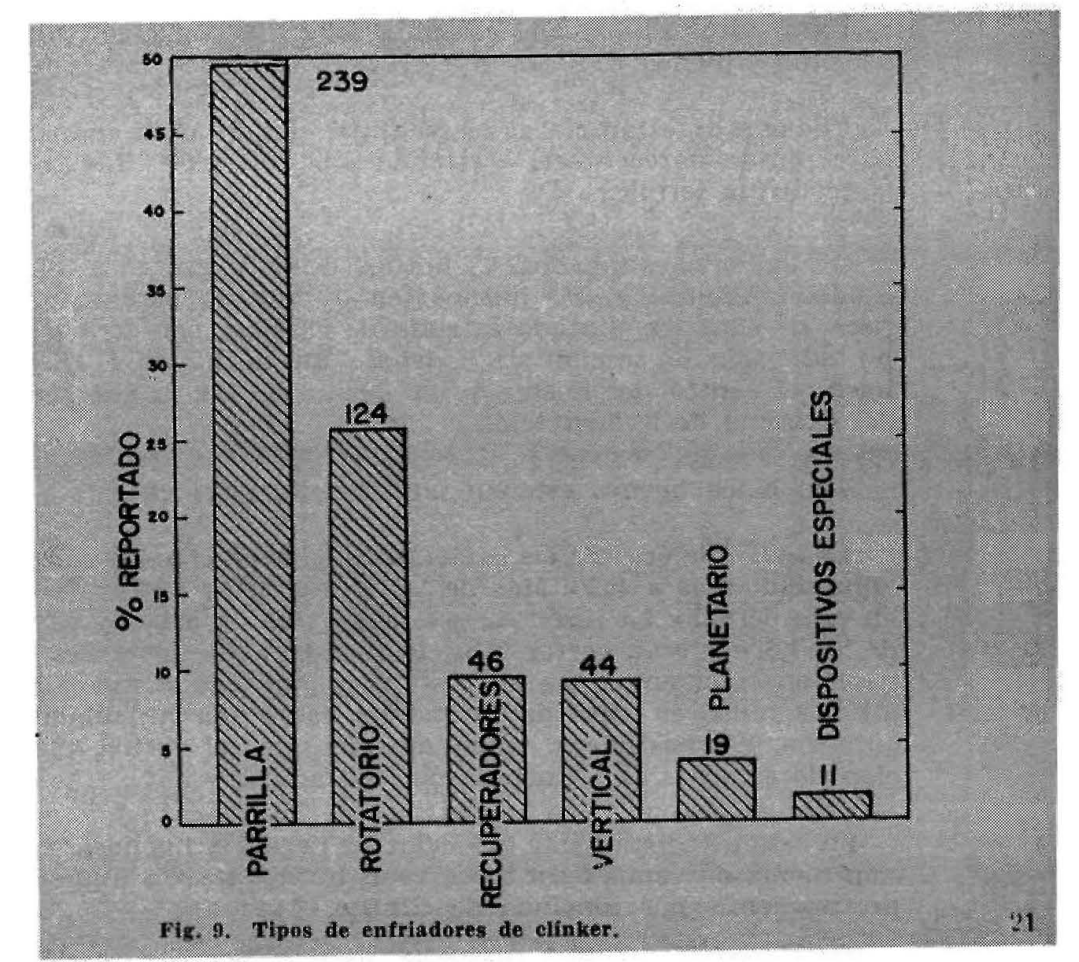




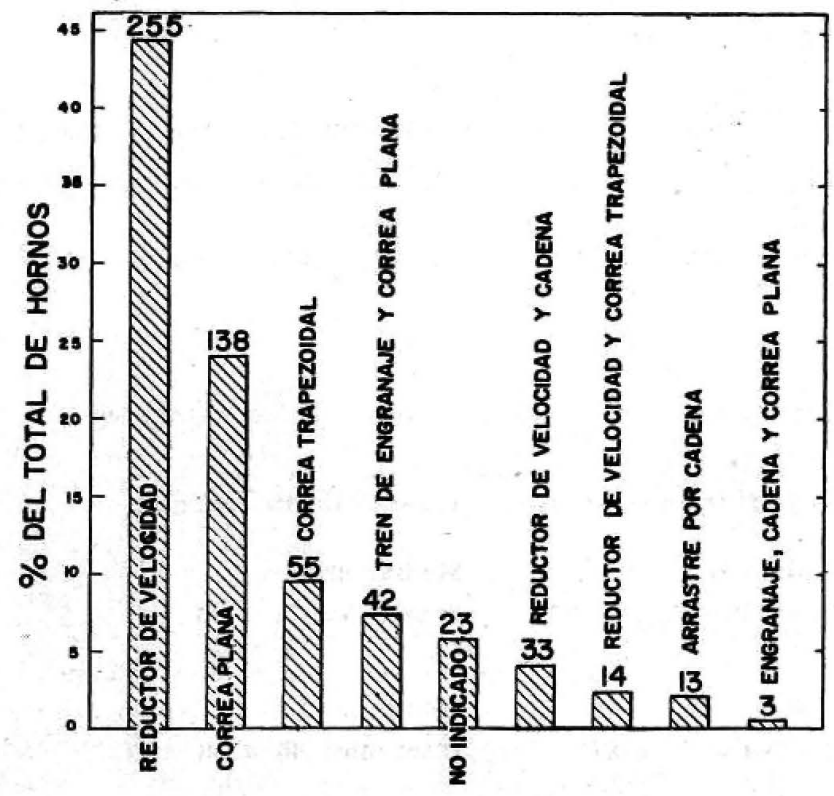

Fig. 10. Tipos de acoplamientos para arrastre del horno.
Los enfriadores planetarios ocupan el $4 \%$ del total, y la temperatura media de salida del clínker en estos aparatos es de $173^{\circ} \mathrm{C}$.

El resto de las instalaciones $(1,7 \%)$ lleva dispositivos especiales, que constan de enfriadores de parrilla cortos seguidos de enfriadores rotatorios, enfriadores planetarios seguidos de enfriadores rotatorios y enfriadores con transportador de arrastre.

Los hornos revisados en este trabajo están arrastrados con motores de corriente continua en la relación $3: 1$, frente al arrastre con corriente alterna. Todos, salvo ocho hornos, emplean un solo motor; estos ocho eran hornos más nuevos y más largos, con $112 \mathrm{~m}$ de longitud como mínimo y motores dobles.

La transmisión entre la fuente de energia y el horno se clasifica en ocho categorías. Con mucho, la más moderna y destacada era el reductor de velocidad que se instaló en el 44,3\% de los hornos. En segundo lugar, y un poco de sorpresa, estaban las correas planas con el $24 \%$. Estos dos inzicados y los restantes se representan en la fig. 10.

Sólo 105 hornos $(18,3 \%)$ tenían arrastre auxiliar: 75 eran con motor de gasolina; 12 eléctricos; 13, eléctricos-diesel; 4, con generador de gas, y 1, eléctrico vapor.

Las velocidades medias de los hornos aumentan al aumentar su longitud, hasta $112 \mathrm{~m}$, para luego bajar ligeramente para hornos mayores de $121 \mathrm{~m}$. El intervalo de velocidad era mayor para los hornos más viejos, más cortos, y por encima de $76 \mathrm{~m}$ este intervalo disminuía rápidamente. El pequeño margen en la velocidad de los hornos más nuevos, más largos, indica que la tendencia moderna de la marcha va hacia velocidades periféricas altas-medias, de 550 a $605 \mathrm{~m} / \mathrm{h}$ de la superficie interior del revestimiento. Esto puede compararse a una velocidad media de $205 \mathrm{~m} / \mathrm{h}$ para hornos de $30,5 \mathrm{~m}$ de longitud o menos.

Todavia no están claras las ventajas de pintar la chapa del horno. Las dos razones más poderosas para pintar, parecen ser la estética y la corrosión. Hay pocas razones para pensar que el pintado afecte la economia térmica.

De los aquí estudiados, 73 hornos estaban pintados total o parcialmente: 43 de vía seca y 30 de vía húmeda. Treinta y seis hornos tenían toda la carcasa pintada, el más largo de ellos de $137 \mathrm{~m}$. En el resto de ellos iba pintada la zona de cocción, con tres excepciones: un horno de $115 \mathrm{~m}$ que llevaba pintada sólo la sección de cadenas; un horno de $117,5 \mathrm{~m}$ con sólo unos $3 \mathrm{~m}$ de longitud pintados hacia el centro del horno, y un horno de $121 \mathrm{~m}$ que tenía pintados unos $53 \mathrm{~m}$ de longitud a partir del extremo de alimentación.

Sólo cinco hornos estaban pintados interiormente.

La «edad de oros de las calderas de recuperación de calor empezó hacia 1916 y acabó en los primeros años siguientes a 1930. Más de las tres cuartas partes de las calderas en funcionamiento se instalaron en este periodo. La razón de este auge y caída bruscos es lógica. Hace cuarenta años, la mayor parte de los hornos eran cortos y las temperaturas de los gases de escape altas. El paso lógico era usar estos gases en crear energía a un costo más bajo que el mercado o donde no era posible adquirirla. En los úlímos años, el costo de la energía comprada ha subido, relativamente, menos que el costo de maquinaria, combustible y mano de obra, de tal forma que, en la mayor parte de los casos, la instalación de calderas se encuentra poco atractiva.

En esta estadística hay 219 calderas para 275 hor nos: 221 de vía seca, y 54 de vía húmeda. La producción media de vapor para todos estos hornos de vía húmeda y seca era de $95 \mathrm{~kg}$ y $113 \mathrm{~kg}$ de vapor, respectivamente, por tonelada de clínker. 